\title{
Consequences of using a new ISRF model for modeling Galactic diffuse gamma-ray emission
}

\author{
F. Niederwanger \\ University of Innsbruck \\ E-mail: felix.niederwanger@uibk.ac.at

\section{O. Reimer}

University of Innsbruck, Institut für Astro- und Teilchenphysik

\section{R. Kissmann}

University of Innsbruck, Institut für Astro- und Teilchenphysik

\section{Popescu}

University of Central Lancashire, School of Physical Sciences and Computing

\section{R. Tuffs}

Max-Planck-Institut für Kernphysik, Astrophysics Department

\begin{abstract}
Galactic diffuse gamma-ray emission is an important constituent among the total observed gamma-ray emission of our Galaxy. Here we present specific developments to accommodate a new Interstellar Radiation Field (ISRF) model for use in the PICARD code for modeling of Galactic cosmic ray propagation and respective gamma-ray emission. On the basis of the new ISRF we provide predictions on Galactic diffuse gamma-ray emission in the $\mathrm{GeV}$ to $\mathrm{TeV}$ energy regime, and compare to a commonly used different ISRF. Emphasis was laid on obtaining robust predictions for observable signatures in the very high energy gamma-ray regime with a special attention to the energy regime for H.E.S.S. One of the most noteworthy results from using the new ISRF indicates increased gamma-ray fluxes in the Galactic Center region between $10 \mathrm{GeV}$ and $10 \mathrm{TeV}$.
\end{abstract}




\section{Introduction}

The Galactic Diffuse emission is a prominent signature of gamma-emission in the regime from few $\mathrm{MeV}$ [1] to the $\mathrm{TeV}$ regime[2, 3, 4], resulting form interactions of cosmic-rays with the interstellar gas distribution and/or radiation field (ISRF). In this work we present insights about consequences for using a recently published new ISRF model[5] in the framework of the cosmic-ray propagation code PICARD [6]. The new ISRF model consists of components describing starlight originating from stellar populations and scattered light by dust), augmented by the Cosmic Microwave Background (CMB) radiation. Its implementation in PICARD allows us to compare gamma-ray emissions using the new ISRF model with that of the GALPROP provided one [7]. This is of particular interest as the new ISRF model features increased intensities in the sub-optical regime and different expectation for gamma-ray emission from Inverse Compton scattering is anticipated [5]. We use the PICARD cosmic-ray transportation code to determine the particle distribution at different locations in our Galaxy to obtain respective gamma-ray spectra and -skymaps.

\subsection{Description of the new ISRF}

The ISRF [5] now accessible in the PICARD cosmic-ray propagation code is given as an axisymmetric intensity distribution at different wavelengths. The frequency binning is logarithmic and chosen in a way to accurately resolve the most relevant spectral features. Starlight from the stellar population (approximately $0.1-10 \mu \mathrm{m}$ ) and the light scattered by the dust in the Galaxy in the range around $1 \mu \mathrm{m}-1 \mathrm{~mm}$ are included separately. The Cosmic Microwave Background (CMB), dominant above $400 \mu \mathrm{m}$, was added for the use in PICARD. The broadband intensity spectrum of the new ISRF is shown in figure 1.

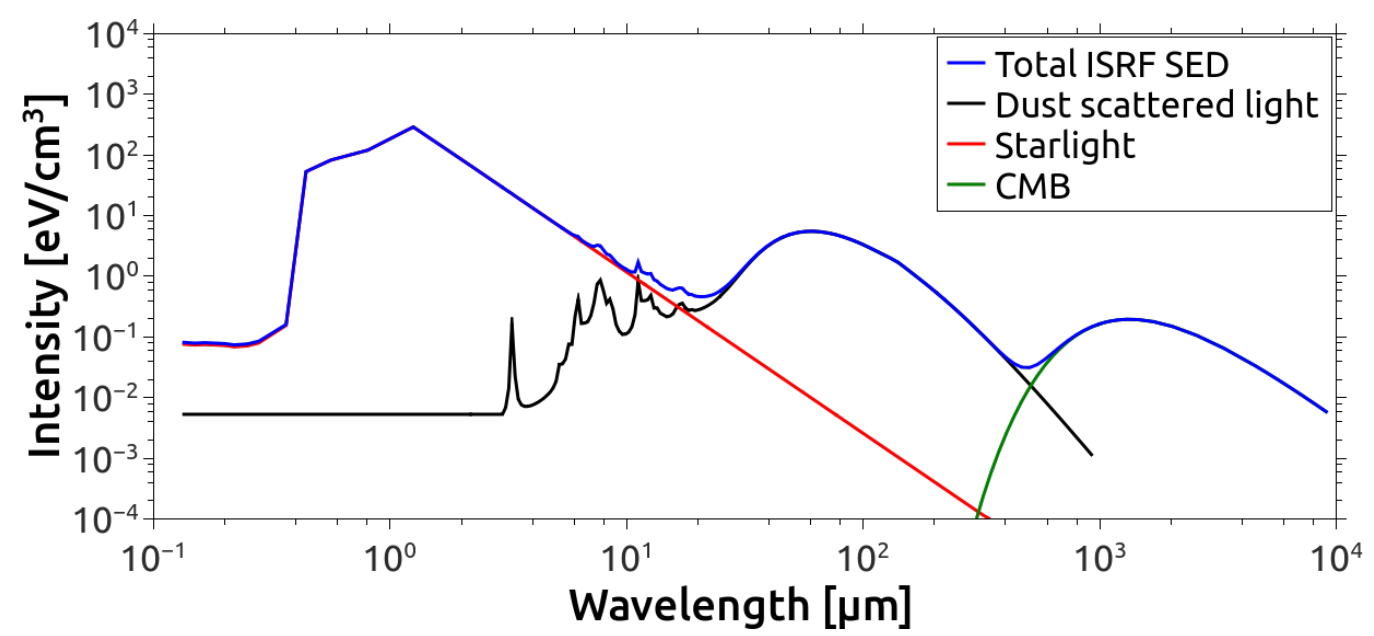

Figure 1: Energy spectrum of the different components in the ISRF at the Galactic center

Within the new ISRF, starlight data are available up to approximately $10 \mu \mathrm{m}$. Beyond that we extrapolate the starlight component with a power law approximation that extends to larger wavelengths. The dust regime is defined for wavelengths between $1 \mu \mathrm{m}$ and $5 \mathrm{~mm}$ and thus resolved accurately in the regime where it is the dominant ISRF component. The contribution of the dust 
component at longer and shorter wavelengths is found by corresponding extrapolation. For wavelengths below $1 \mu \mathrm{m}$ or above $400 \mu \mathrm{m}-800 \mu \mathrm{m}$, the dust regime is at least one order of magnitude below the governing component and thus negligible.

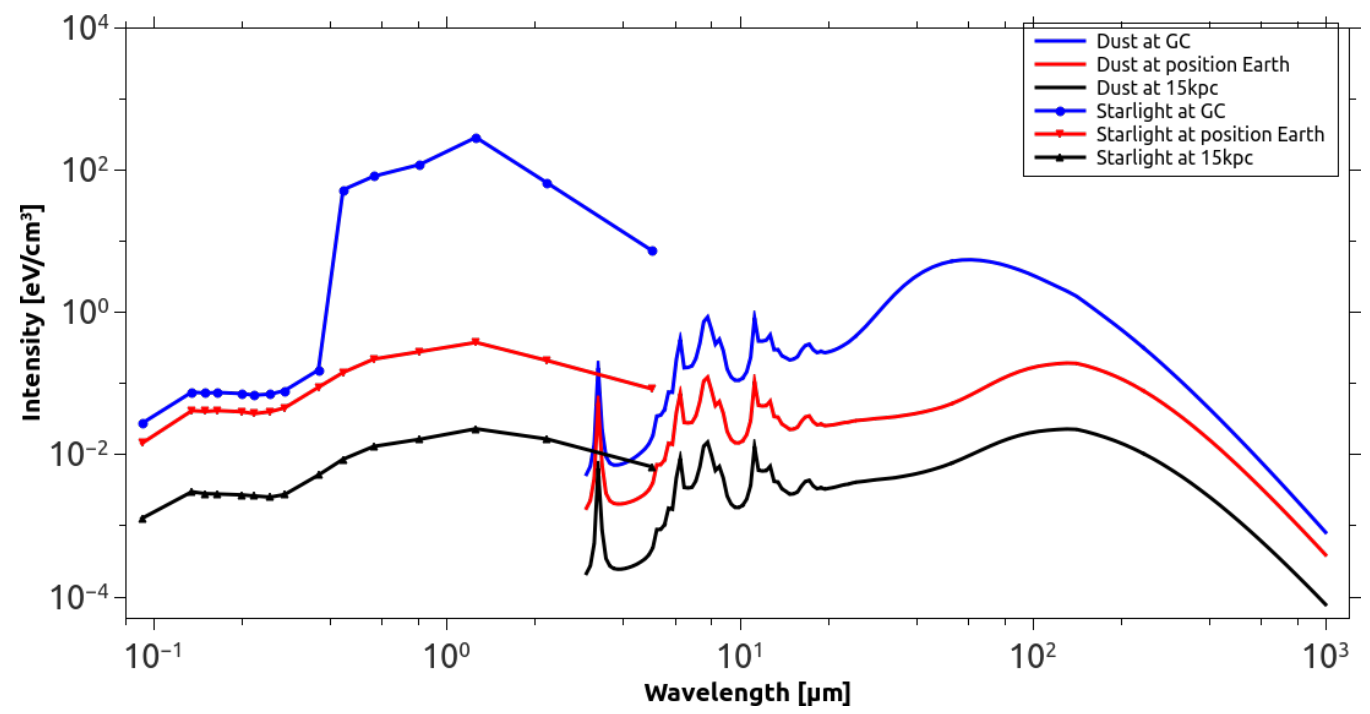

Figure 2: Starlight and dust component of the new ISRF at different $(R, z)$ locations within the Galaxy

The starlight and dust components at different locations in the Galaxy are shown in figure 2. The transition from UV to optical light of the stellar component at roughly $0.4 \mu \mathrm{m}$ has a discontinuity at the Galactic Center but is smooth elsewhere. The starlight component is defined over 15 frequency bins while the dust component has 120. The dust component has much more frequency bins in order to resolve the given spectral features accurately (see figure 2).

In comparison to the GALPROP ISRF [7], the ISRF intensities in the sub-optical and the spectral resolution are significantly increased with the new ISRF model. In comparison to our study in [8], we are now using an improved ISRF model. Further the internal frequency resolution of the ISRF was increased due to technical improvements on the code. In the proceeding we were using the same frequency binning as the GALPROP ISRF model. Nevertheless, the differences because of the higher resolution are rather small in respect to the differences between the ISRF intensities.

\subsection{PICARD - The propagation framework}

PICARD is a cosmic-ray propagation code that allows also for spatial and spectral predictions of gamma-ray emission intensities [6]. The propagation of cosmic rays is computed by solving the cosmic-ray transport equation by assuming a steady-state solution. The transport physics can be defined via a set of parameters. Here, we have computed the comic-ray distribution and gamma-ray emission maps for an axisymmetric model of our Galaxy for the two ISRFs. The source distribution for the axisymmetric model is based on the radial distribution of pulsars within the Galaxy by Yusifov and Kücük [9].

For this proceeding we are focussing exclusively on the IC emission component given its direct connection to the ISRF. Effects relating to Bremsstrahlung are negligible for the energies discussed 
here.

\section{Comparison between different ISRF models}

We compare the new ISRF model with the recent GALPROP ISRF model[7] and notice three principal differences:

- Increased sub-optical ISRF intensities $(2 \mu \mathrm{m}-100 \mu \mathrm{m})$

- Higher intensities towards the Galactic center, Galactic Bulge, and the Galactic plane

- Increased frequency resolution in the dust dominated regime

The two ISRF models agree well with each other regarding principal spectral shape (Fig. 3). The starlight component varies up to more than an order of magnitude in the Galactic Center. Differences are also prominent in the dust component in the outside regions. Spectral features in the dust regime are similar, although with comparably higher intensity in [5]. In the Galactic Center, this discrepancy amounts to more than an order of magnitude from from $0.4 \mu \mathrm{m}$ to the high dust regime at $40 \mu \mathrm{m}$. The new ISRF has an underabundance of UV photons below $40 \mu \mathrm{m}$.

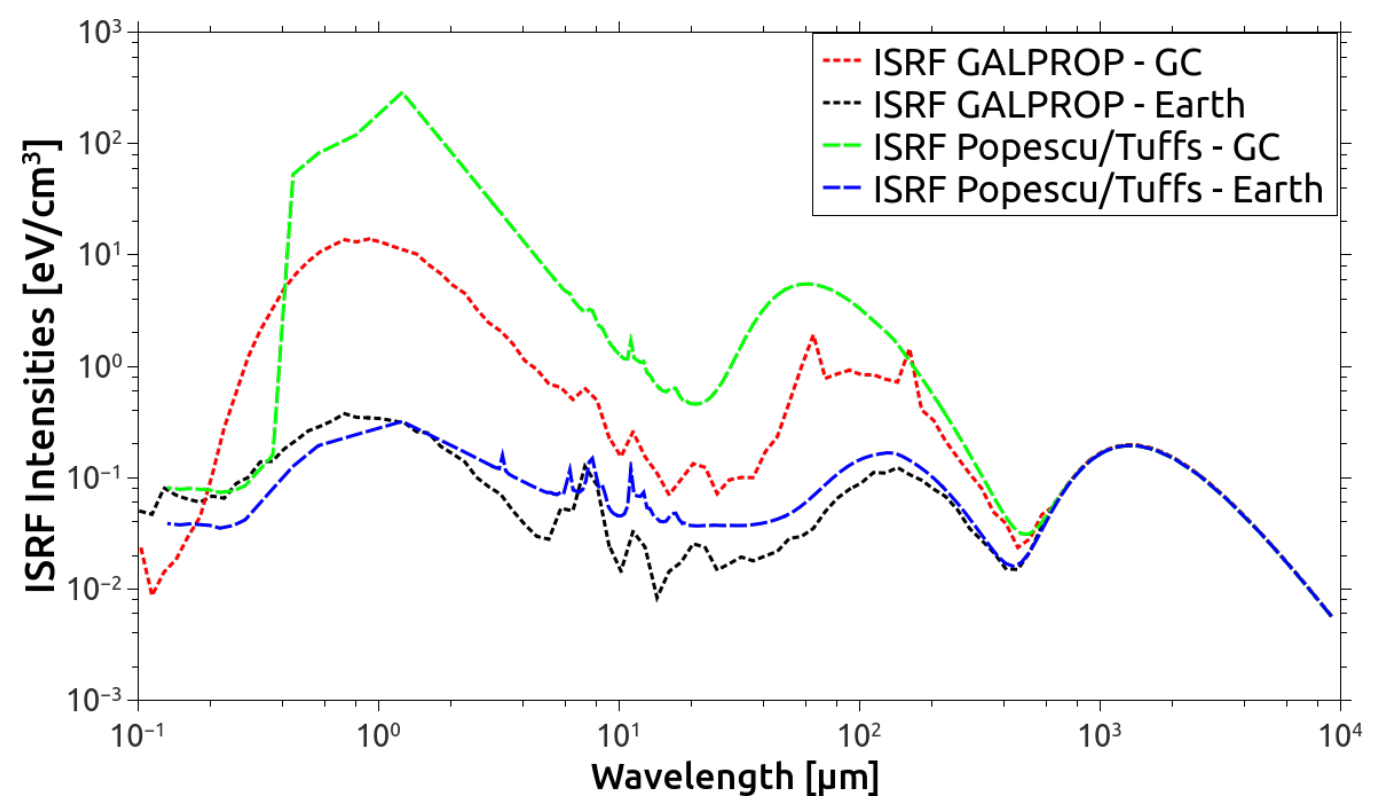

Figure 3: Broadband intensity spectrum of the two ISRF models at the position of the Galactic Center and Earth, respectively

\subsection{Differences regarding cosmic-ray electron spectra}

We now study the CR particle distributions that affect IC predictions when using different ISRFs. This concerns the primary CR electron spectrum and the secondary leptons produced by $\mathrm{CR}$ interactions. We first look at the electron loss rates. IC energy loss rates scale linearly with the corresponding ISRF intensities. However, the electron loss rate depends on the convolution of the photon number density with the differential Klein-Nishina cross section over all photon 
energies[10].

Indicated by the different intensities in the ISRF at the Galactic Center also the energy loss rates are about an order of magnitude larger when using the ISRF of [5]. In consequence, IC is the dominant energy loss rate for an extended energy range, This is particularly evident towards higher energies, when synchrotron losses become dominant at $100 \mathrm{TeV}$ for [5], instead at $1 \mathrm{TeV}$ for [7].

Since electrons lose their energy more efficiently in the presence of a higher ISRF intensity, an increased IC energy loss rate yields reduced high-energy CR electron fluxes and accordingly larger gamma-ray flux.

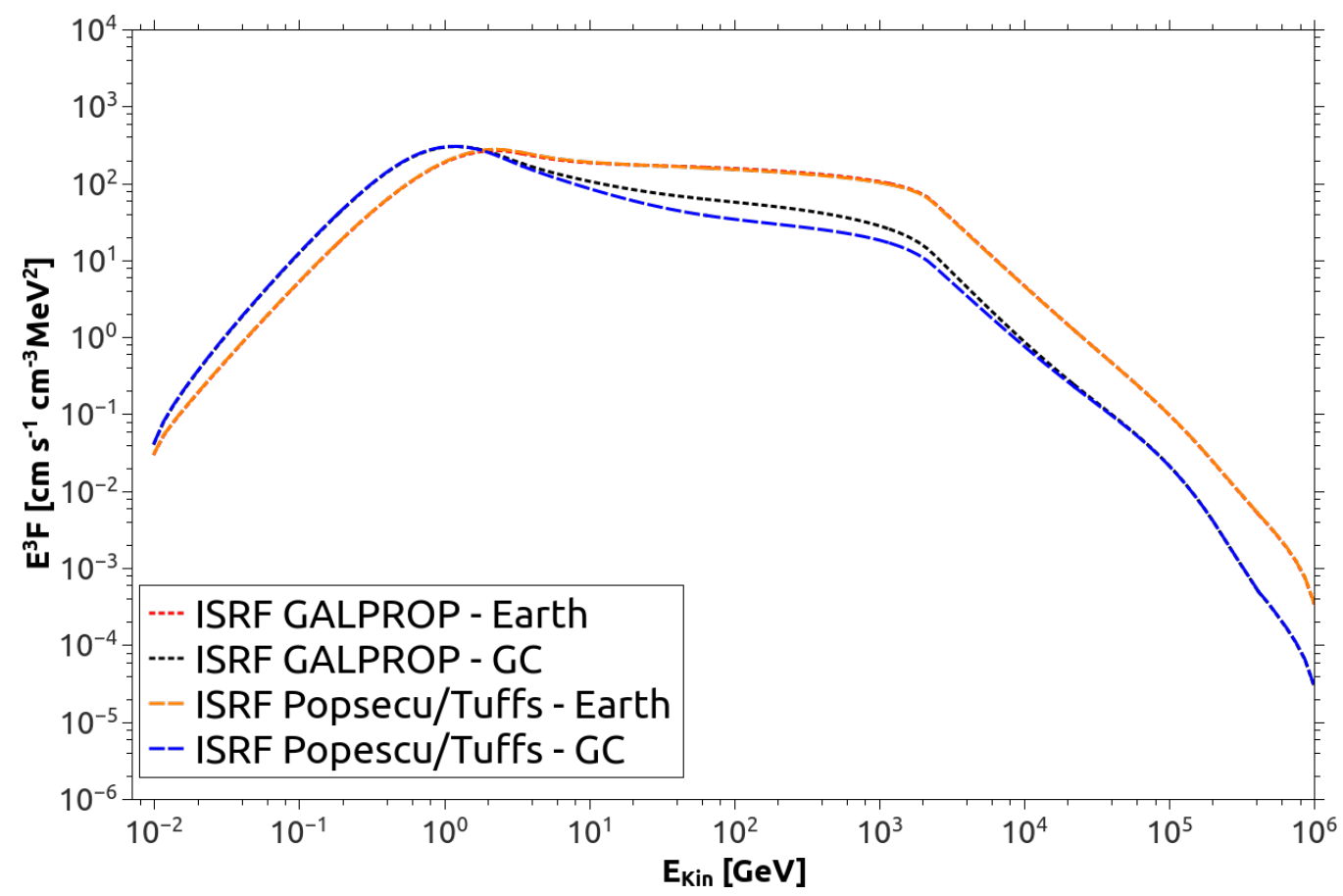

Figure 4: $E^{3} F$ electron spectrum at the Galactic Center and position Earth for both ISRF model

Figure 4 shows the resulting electron spectrum at the Galactic Center and at position Earth. In the Galactic Center, where the discrepancy between the two ISRFs is largest, the electron flux between $10 \mathrm{GeV}$ and $10 \mathrm{TeV}$ is reduced by $32 \%$ (integrated flux) due to the increased electron losses. Below $10 \mathrm{GeV}$ the $\mathrm{CR}$ electron spectrum in the Galactic Center compares rather well between the different ISRF models.

For our models, we get a spectral index of the electron spectrum in the Galactic Center between 10 and $500 \mathrm{GeV}$ of 3.25 , in comparison to that at Earth of 3.08 in contrast to a spectral index of $3.18 \pm 0.05$ above $30 \mathrm{GeV}$ as suggested by PAMELA [11], 3.04 above $20 \mathrm{GeV}$ indicated by Fermi-LAT[12] (up to $1 \mathrm{TeV}$ ) or $3.05 \pm 0.02$ suggested by the H.E.S.S. collaboration, with a cutoff at $2.1 \pm 0.3 \mathrm{TeV}[13]$. The differences in the measured spectral indices of the electron spectrum show the importance of cosmic-ray propagation models for the predictions of realistic gamma-ray skymaps. This contrasts mildly to the spectral index of 3 used throughout the Galaxy in [5].

\subsection{Differences regarding high-energy gamma-ray spectra}

We expect largest gamma-ray intensity when the electron flux folded with the electron loss 
rates is largest. The work of [5] underlines already the importance of the ISRF for IC gamma-ray emission assuming a homogeneous electron spectrum.

Figure 5 shows the gamma-ray spectra at the Galactic Center for all relevant gamma-ray production channels. The general shape of the resulting total diffuse gamma-ray emission shows an increase between $10 \mathrm{GeV}$ and $1-10 \mathrm{TeV}$ when using the new ISRF model. This peak value for this increase in the Galactic center region $\left(|r| \leq 3^{\circ}\right)$ is $16 \%$ at $285 \mathrm{GeV}$. The IC component is increased globally without showing additional features. Consequently we observe a slightly higher total gamma-ray flux in this energy regime. At energies above $10 \mathrm{TeV}$, IC emission drops below $20 \%$ because of the reduced Klein-Nishina cross section towards higher energies. Thus the relevant regime for the production of diffuse IC gamma-ray emission is below $10 \mathrm{TeV}$ and the influence of the new ISRF model is dominant in the regime between $10 \mathrm{GeV}$ and $10 \mathrm{TeV}$.

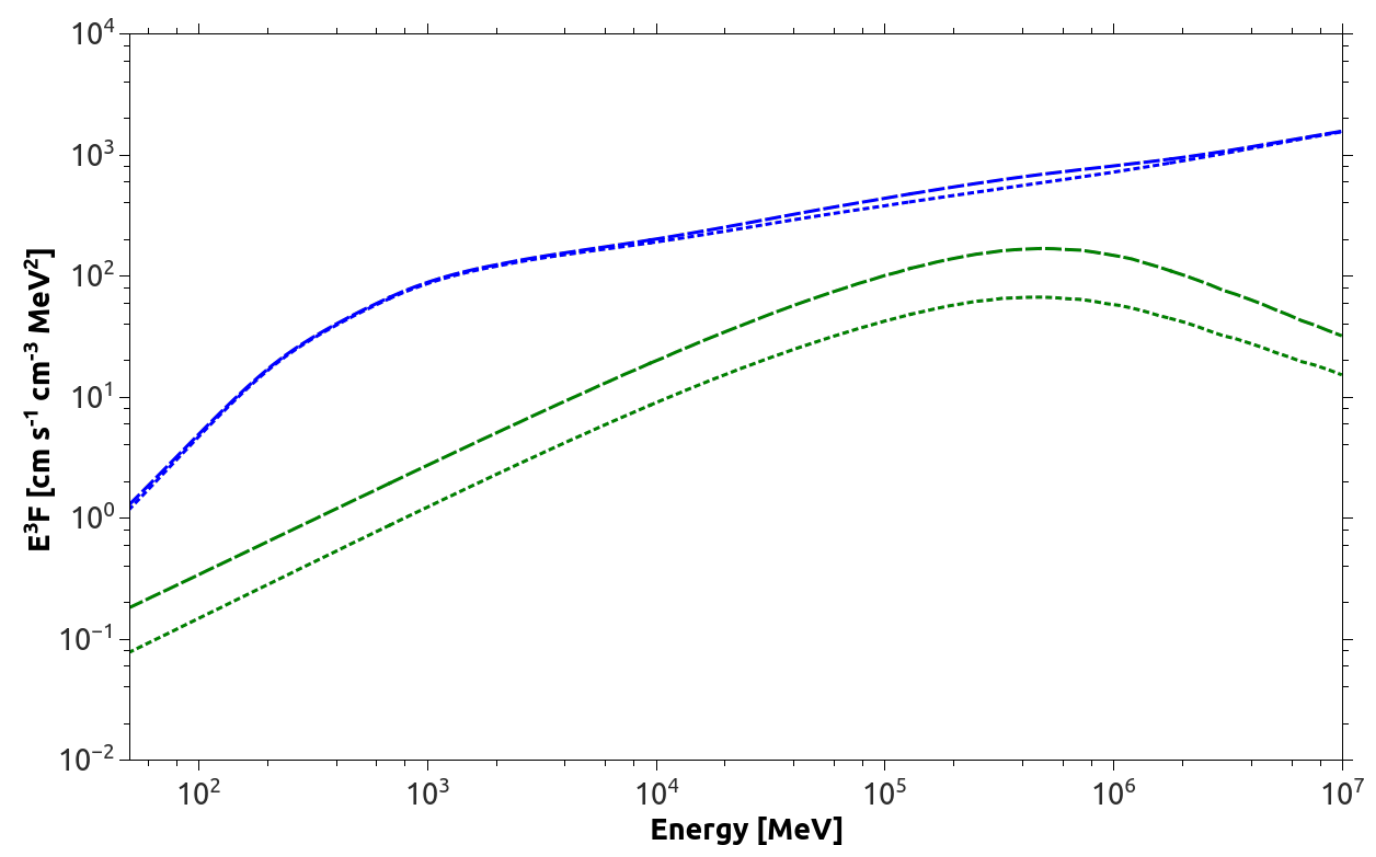

Figure 5: IC Gamma-ray spectrum at the Galactic center for the two ISRF models. Blue - Total DGE, Green - IC gamma-rays only. Dashed line - Popsescu/Tuffs ISRF, Dashed line - GALPROP ISRF model

\section{Differences regarding the high-energy gamma-ray skymaps}

Fig. 6 shows latitude and longitude profiles at different energies for the ISRF models [5] and [7], respectively. For the new model we find increased IC intensities in all directions with a particularly enhanced flux towards the Galactic center and in the Galactic plane, reflecting the higher ISRF inten- sities there. This behaviour is present for all energies, but being largest in the range $10 \mathrm{GeV}-10 \mathrm{TeV}$ as discussed in section 2.2.

\section{Conclusion}

In contrast to the ISRF GALPROP model [7], the new ISRF model [5] features significantly higher intensities in the Galactic Center. The correspondingly higher IC losses cause a decreased 

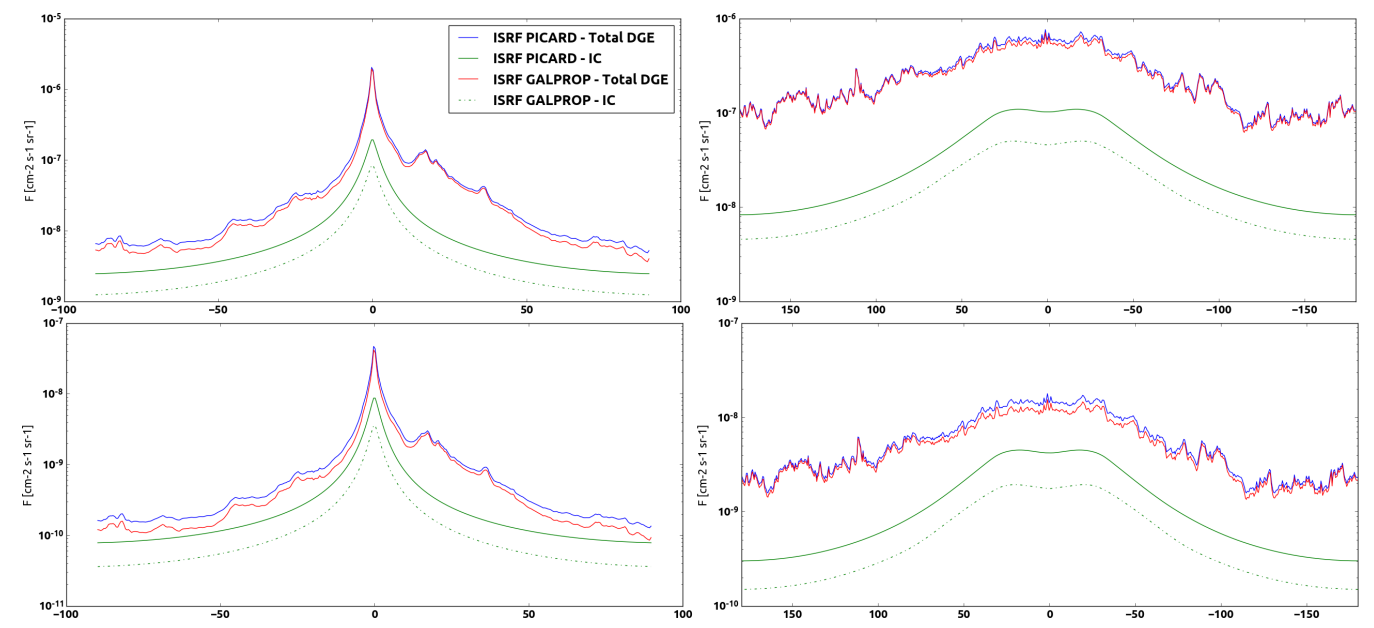

Figure 6: Latitude (left) and longitude (right) profiles for diffuse gamma-ray emission between $10 \mathrm{GeV}$ and $10 \mathrm{TeV}$ (upper panel) and between $100 \mathrm{GeV}$ and $1 \mathrm{TeV}$ (lower panel), where the effect on IC emission is largest.

flux of CR electrons between $1 \mathrm{GeV}$ and $100 \mathrm{TeV}$ in the GC, while the electron spectra at Earth show no significant differences for the two ISRF models. Especially towards the Galactic Center and also within the Galactic plane, this new ISRF leads to a considerably higher contribution from IC scattering to the total gamma-ray emission. This increase is significant when considering CR propagation on deca-parsec-scales. The impact of the new ISRF model on the diffuse gamma-ray emission is consequently the increase of IC gamma-ray emission, also visible as a increased total gamma-ray flux at energies between $10 \mathrm{GeV}$ and $10 \mathrm{TeV}$.

\section{References}

[1] M. Ackermann, M. Ajello, W. B. Atwood, et al. Fermi-LAT Observations of the Diffuse $\gamma$-Ray Emission: Implications for Cosmic Rays and the Interstellar Medium. Astroparticle Journal, 750:3, May 2012.

[2] A. A. Abdo, B. Allen, T. Aune, et al. A Measurement of the Spatial Distribution of Diffuse TeV Gamma-Ray Emission from the Galactic Plane with Milagro. The Astrophysical Journal, 688(2):1078, 2008.

[3] A Abramowski, F Aharonian, F Ait Benkhali, et al. Diffuse galactic gamma-ray emission with hess. Physical Review D, 90(12):122007, 2014.

[4] M Amenomori, S Ayabe, SH Cui, et al. Observation of multi-tev diffuse gamma rays from the galactic plane with the tibet air shower array. The Astrophysical Journal, 580(2):887, 2002.

[5] C. C. Popescu, R. Yang, R. J. Tuffs, et al. A radiation transfer model for the Milky-Way: I. Radiation fields and application to High Energy Astrophysics. ArXiv e-prints, May 2017.

[6] R. Kissmann. PICARD: A novel code for the Galactic Cosmic Ray propagation problem. Astroparticle Physics, 55:37-50, March 2014.

[7] T. A. Porter, I. V. Moskalenko, A. W. Strong, et al. Inverse Compton Origin of the Hard X-Ray and Soft Gamma-Ray Emission from the Galactic Ridge. The Astrophysical Journal, 682:400-407, July 2008. 
[8] F. Niederwanger, O. Reimer, R. Kissmann, et al. The use case of a new ISRF on diffuse gamma-ray emission models. In AIP Conference Proceedings, volume 1792, page 070015. AIP Publishing, 2017.

[9] I. Yusifov and I. Küçük. Revisiting the radial distribution of pulsars in the Galaxy. A\&A, 422:545-553, August 2004.

[10] Frank C Jones. Inverse compton scattering of cosmic-ray electrons. Physical Review, 137(5B):B1306, 1965.

[11] O. Adriani, G. C. Barbarino, G. A. Bazilevskaya, et al. Cosmic-Ray Electron Flux Measured by the PAMELA Experiment between 1 and 625 GeV. Phys. Rev. Lett., 106:201101, May 2011.

[12] Aous A Abdo, Markus Ackermann, Marco Ajello, et al. Measurement of the cosmic ray e++ espectrum from $20 \mathrm{GeV}$ to $1 \mathrm{TeV}$ with the Fermi Large Area Telescope. Physical Review Letters, 102(18):181101, 2009.

[13] F Aharonian, AG Akhperjanian, U Barres De Almeida, et al. Energy spectrum of cosmic-ray electrons at tev energies. Physical Review Letters, 101(26):261104, 2008. 